\title{
PERFORMANCE ANALYSIS OF CLUSTER BASED ROUTING SCHEME IN WIRELESS SENSOR NETWORK
}

\author{
Hrituparna Paul ${ }^{1}$ and Prodipto Das ${ }^{2}$
}

\begin{abstract}
Wireless Sensor Networks have formed new opportunities across the variety of human efforts including engineering design, forest fire tracking, and battlefield surveillance. It is a group of sensor nodes that comprised of sensing, computing, and communication elements that gives an administrator the ability to observe and react to events and phenomena in a specified environment. Minimizing Energy consumption is considered as one of the most important principles in the development of routing protocols for Wireless Sensor Networks (WSN). This research paper describes the characteristics of hierarchical routing scheme for sensor network Low Energy Adaptive Clustering hierarchy (LEACH) and Location Aware Cluster Based Routing Protocol (LACBRP) based on the performance matrices like Energy Values, Latency Values, Packet Delivery Ratio and Residual Energy Values. This comparative study proves that LACBRP performs well than LEACH routing scheme.

Keyword: WSN, Energy Values, Latency Values, Packet Delivery Ratio, Residual Energy Values
\end{abstract}

\section{INTRODUCTION}

Wireless sensor network (WSN) is a densely deployed collection of a large number of selforganising wireless sensor nodes. It has a limited energy resource with a base station to collect and process the data from sensor nodes.WSN is a compilation of sensor nodes, capable of collecting information from their environment. These nodes have the capability of sensing, computing, and wireless communicating. Various monitoring tasks such as search, rescue, disaster relief, target tracking and a number of tasks in smart

environments are performed by WSN. In many such tasks, Clustering is one of the fundamental challenges in wireless sensor network. Wireless Sensor Networks is a focused wireless network made up of a large number of sensors and at least one base station. Energy saving is also a crucial issue in designing the wireless sensor networks [1]. In order to maximize the lifetime of sensor nodes, it is preferable to distribute the energy dissipated throughout the wireless sensor network. So it is essential to design effective and energy aware protocols in order to enhance the network lifetime. In this paper, Low Energy Adaptive Clustering Based Routing (LEACH), Base station positioning Algorithm (BSP), Energy-Balanced Routing Method (BEC) and Location

\footnotetext{
${ }^{1}$ Assam University, INDIA

${ }^{2}$ Assam University, INDIA
} 
Aware Cluster Based Routing (LACBRP) which are the part of network structure protocol is carried out. Energy consumption and network life time has been considered as the major issues wireless sensor network (WSN) requires a huge breadth of knowledge from an enormous variety of disciplines, so its study becomes challenging [1].

This paper is categorized as follows. Section I presents the Introduction. Section II provides the overview of Routing protocols. Section III describes the simulation environment .Section IV presents the Experimental results and Section V concludes the paper.

\section{OVERVIEW OF ROUTING PROTOCOLS}

LEACH: It is Dynamic Hierarchical Routing for sensor network, called Low Energy Adaptive Clustering Hierarchy (LEACH). A routing protocol is considered adaptive if certain system parameters can be restricted in order to adapt to current network conditions and available energy levels. LEACH randomly selects a few sensor nodes as cluster heads $(\mathrm{CHs})$ and rotates this role to evenly share out the energy load among the sensors in the network. In LEACH, the CH nodes compress data arriving from nodes that belong to the respective cluster, and send an aggregated packet to the BS in order to reduce the amount of information that must be transmitted to the BS. LEACH uses a TDMA/code division multiple access (CDMA) MAC to reduce inter cluster and intra-cluster collisions. However, data collection is centralized and performed periodically. Therefore, this protocol is most appropriate when there is a need for constant monitoring by the sensor network. A user may not need all the data immediately. Hence, periodic data transmissions are unnecessary, and may drain the limited energy of the sensor nodes. After a given interval of time, randomized rotation of the role of $\mathrm{CH}$ is conducted so that uniform energy dissipation in the sensor network is obtained. The operation of LEACH is separated into two phases, the setup phase and the steady state phase. In the setup phase, the clusters are organized and $\mathrm{CHs}$ are selected. In the steady state phase, the actual data transfer to the BS takes place. The duration of the steady state phase is longer than the duration of the setup phase in order to minimize overhead. During the setup phase, a predetermined fraction of nodes, $p$, elect themselves as $\mathrm{CHs}$ as follows. A sensor node chooses a random number, $r$, between 0 and

1. If this random number is less than a Threshold value, $\mathrm{T}(\mathrm{n})$, the node becomes a $\mathrm{CH}$ for the current round. The threshold value is calculated based on an equation that incorporates the desired percentage to become a $\mathrm{CH}$, the current round, and the set of nodes that have not been selected as a $\mathrm{CH}$ in the last (1/p) rounds denoted as $\mathrm{G}$. it is given by:

\section{T $\quad(n)=(p /(1-p(\bmod (1 / p))))$ if $n € G$}

where $G$ is the set of nodes that are involved in the $\mathrm{CH}$ election. All elected $\mathrm{CHs}$ broadcast an advertisement message to the rest of the nodes in the network that they are the new CHs. All the non $-\mathrm{CH}$ nodes, after receiving this advertisement, decide on the cluster to which they want to belong. This decision is based on the signal strength of the advertisement. The non- $\mathrm{CH}$ nodes inform the appropriate $\mathrm{CHs}$ that they will be a member of the cluster. After receiving all the messages from the nodes that would like to be included in the cluster and based on the number of nodes in the cluster, the $\mathrm{CH}$ node creates a TDMA schedule and assigns each node a time slot when it can transmit. This schedule is broadcast to all the nodes in the cluster. During the steady state phase, the sensor nodes can begin sensing and transmitting data to the $\mathrm{CHs}$. The $\mathrm{CH}$ node, after receiving all the data, aggregates it before sending it to the BS. After a certain time, which 
is determined a priori, the network goes back into the setup phase again and enters another round of selecting new $\mathrm{CHs}$. Each cluster communicates using different CDMA codes to reduce interference from nodes belonging to other clusters.

Limitation: It is not applicable to networks deployed in large regions. It also assumes that nodes always have datal to send, and nodes located close to each other have correlated data. It is not obvious how the number of predetermined $\mathrm{CHs}(p)$ is going to be uniformly distributed through the network.

BSP [7]: Base Station Positioning Routing Algorithm proposed by Tripathy et al. [7]. The objective of this algorithm is to minimise the overall energy consumption in a WSN and find such a base station location

.Considering some nodes to be far enough to use a different path loss model for their signals to the base station, here proposed algorithm considers two categories of nodes and hence two different path loss models based on their distance from the base station. The optimal location of a base station can be analysed with respect to minimum energy expenditure or maximum lifetime of a sensor network.

BEC [8]: Energy-Balanced Routing Method Based on Forward-Aware Factor proposed by Zhang et al.

[108]. This algorithm quantify the forward transmission area, define forward energy density, which constitutes forward-aware factor with link weight, and propose a new energy-balance routing protocol based on forward-aware factor (FAF-EBRM). Thus balances the energy consumption, prolongs the function lifetime.

LACBRP [2]: The location aware cluster based routing uses three phases in wireless sensor networks. In the first phase, the location information of each sensor node is computed by using the localization algorithm such as Trilateration, Triangulation etc; in the second phase, the sensor nodes are clustered to minimize the residual energy and maximize the network performance then the Cluster head is elected based on the minimum distance between the cluster node's and the centroid; in the third phase, Routing takes place between the cluster head and the cluster members and also between the cluster head and the base station.

A. Location of Sensor node: The location information of each sensor node should be known to form a cluster in the wireless sensor network. The nodes which are deployed in the sensor network, knows their location information. The coordinates (xi, yi) of each sensor node are used to estimate the distance between two sensor nodes. Trilateration is a geometric principle which is used to find a location, if their distances from other nodes are known. It computes a node's position via the intersection of three circles. To calculate the unknown node's location, trilateration uses the known locations of two or more reference points, and the measured distance between the unknown node and each reference point. 
Performance Analysis of Cluster Based Routing Scheme in Wireless Sensor Network 294

The distance between reference nodes is computed by using this formula:

Distance $=\sqrt{\left(x_{2}-x\right)_{1}^{2}+(y-y)^{2}}$

Here, $\left(\mathrm{x}_{1}, \mathrm{y}_{1}\right)$ and $\left(\mathrm{x}_{2}, \mathrm{y}_{2}\right)$ are the coordinates of the reference node.

The new coordinate is computed by using this formula,

$$
\begin{gathered}
x=\frac{\left.y_{(} y_{a}-y_{b}\right)}{\left(x_{b}-x_{c}\right)} \\
y=\frac{v_{b}\left(x_{b}-x_{c}\right)-v_{a}\left(x_{b}-x_{a}\right)}{\left(y_{a}-y_{b}\right)\left(x_{b}-x_{a}\right)-\left(y_{c}-y_{b}\right)\left(x_{b}-x\right)}
\end{gathered}
$$

Where,

$x, y$ is the new coordinate.

$V_{a}$ and $V_{b}$ are the relative distance between two spheres.

$x_{a}, x_{b}, x_{c}$ and $\mathrm{y}_{a}, y_{b}, y_{c}$ are the $\mathrm{x}$ and $\mathrm{y}$ coordinates of three reference points.

\section{B. Cluster Formation:}

In the first step, randomly select c cluster head with their xi, yi coordinates. Then calculate the distance between each sensor node and the randomly selected cluster head and also get the energy of each node.

\section{Cluster head selection:}

After the formation of cluster, re-compute the centroid of the clusters resulting from the calculated distance. Calculate the centroid point of each cluster in the wireless sensor network. The centroid point is the new coordinate which is not equal to any position of sensor node in the wireless sensor network.

\section{SIMULATION ENVIRONMENT:}

The aim of this simulation is to evaluate the characteristics of hierarchical routing scheme for sensor network Low Energy Adaptive Clustering hierarchy (LEACH), Base Station Positioning Routing Algorithm (BSP), Energy-Balanced Routing Method (BEC) and Location Aware Cluster Based Routing Protocol (LACBRP) based on the performance matrices like Energy Values, Latency Values. This simulation of Clustering is done in ns2. The Number of nodes considered here is 40 
TABLE 1: SIMULATION PARAMETERS:

\begin{tabular}{|c|c|}
\hline Parameter & Value \\
\hline Number of nodes & 40 nodes \\
\hline Mac Layer Type & 802.11 \\
\hline Topology size & $800 \times 600(\mathrm{mxm})$ \\
\hline Routing protocol & AODV \\
\hline Propagation model & TwoRayGround \\
\hline Energy model & Energy Model \\
\hline
\end{tabular}

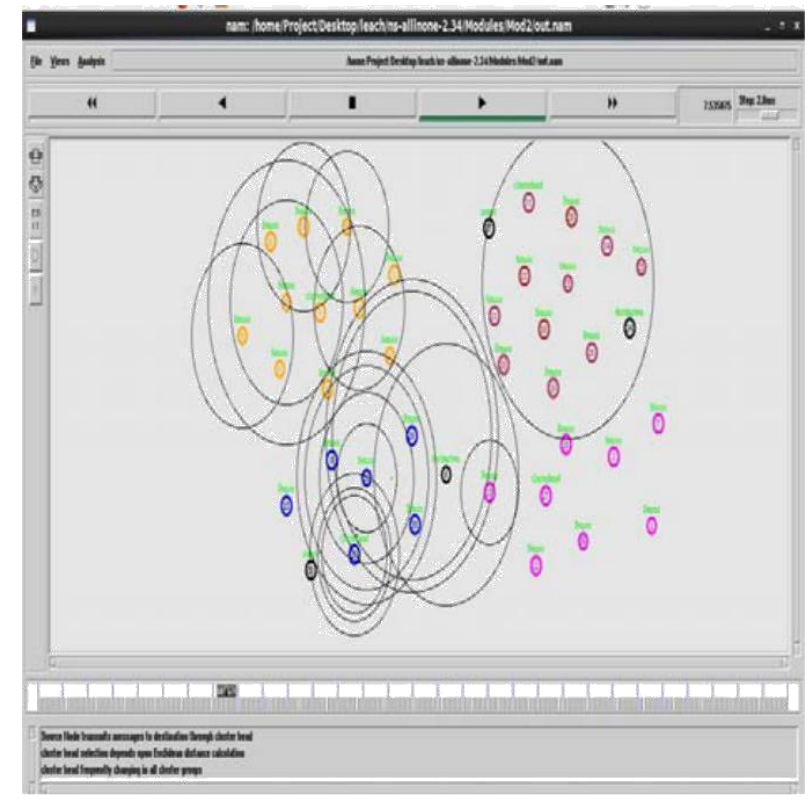

Figure. 1: Layout of LEACH Simulation

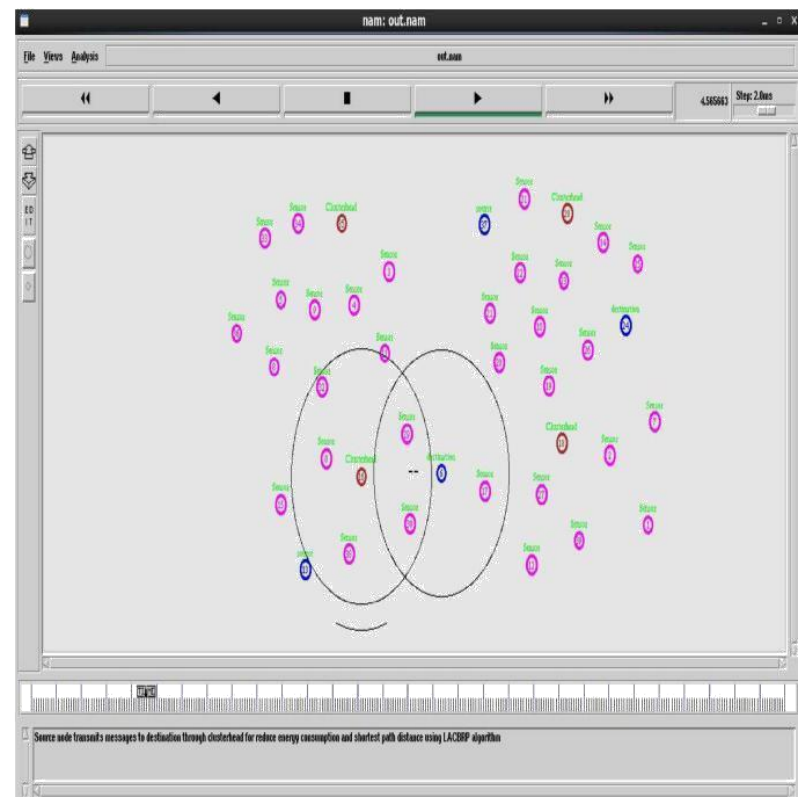

Figure. 2: Layout of LACBRP Simulation 


\section{RESULTS AND PERFORMANCE ANALYSIS}

In this paper we have compared the performance of LEACH and LACBRP based on various factors. Now an analysis is made on the achieved results based on various factors like percentage of Energy Values, Latency Values, Packet Delivery Ratio and Residual Energy Values during different mobility conditions.

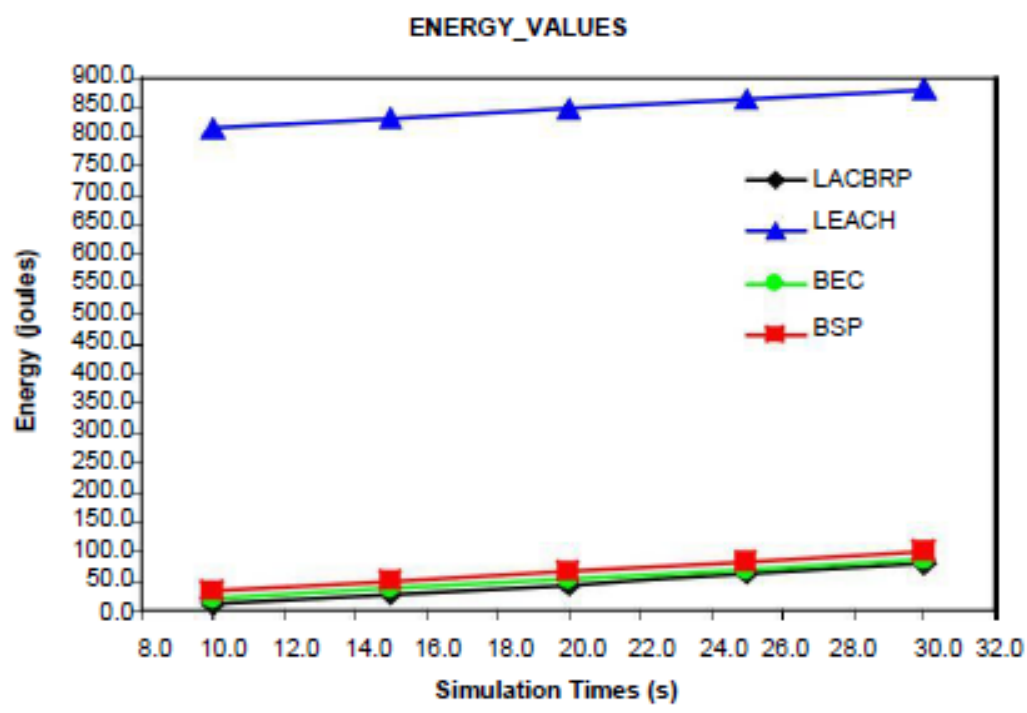

Figure 3: Simulation Time Vs Energy Values

The energy consumption value at $10 \mathrm{sec}$ simulation time is observed to be 814.754 for LEACH, 33.184 for BSP, 18.562 for BEC and 8.777 for LACBRP respectively and at an interval of 30 sec, the values are $881.358,98.808,84.820$ and 80.417 respectively. From this values it can be clearly observed that the LACBRP routing scheme consumes the lowest energy in comparison to the other routing scheme i.e LEACH routing scheme, Base Station Position routing scheme and Energy-Balanced routing scheme. 


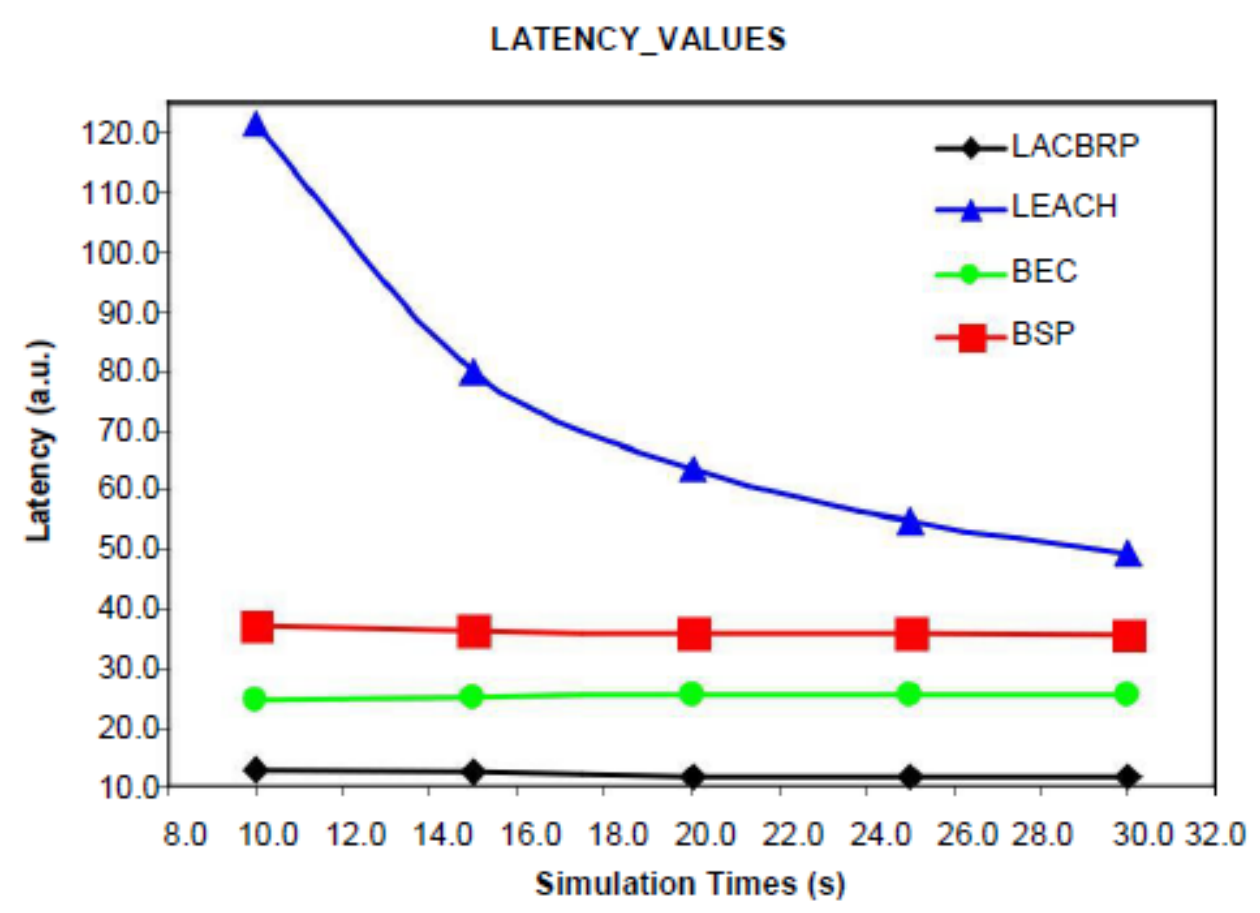

Figure 4: Simulation Time Vs Latency Values

Figure 4 shows the variation of Latency values with time from $10 \mathrm{sec}$ to $30 \mathrm{sec}$ at an interval of 5 secs. The Latency Values is observed to be 121.494 for LEACH, 37.215 for BSP, 24.8182 for BEC and 12.7867 for LACBRP respectively and at an interval of $30 \mathrm{sec}$, the values are 49.2427, $35.5849,25.4303$ and 11.5275 respectively. From these values it can be clearly observed that the LACBRP routing scheme respond at a faster rate means it has the minimum latency value in comparison to the other routing scheme i.e LEACH routing scheme, Base Station Position routing scheme and Energy-Balanced routing scheme. 


\section{CONCLUSIONS}

In this work, a detail simulation survey of the different clustering algorithms considering Energy Consumption Value, Latency Value, are being presented for energy constrained wireless sensor network. Simulation results are discussed to describe the effect of $\mathrm{CH}$ selection and the size of the cluster based on the parameters like Energy Consumption Value, Latency Value,. The Algorithm i.e LACBRP should be able to adapt the network clusters to maintain network connectivity while reducing the wasteful resources associated with periodic re-clustering and also performs better and provides the high performances like different routing parameter i.e Energy Consumption Value, Latency Value, in ns-2 in different routing scheme such as LEACH routing scheme, Base Station Position routing scheme and Energy-Balanced routing scheme.

Here we compare the performance of LACBRP protocol with the existing clustering protocols LEACH, BSP and BEC. Simulation Result illustrate that proposed LACBRP outperforms than LEACH but has almost similar performance to BSP and BEC protocols in terms of network energy consumption. From the Figure 4 it can clearly observed that the LACBRP routing scheme respond at a faster rate means it has the minimum latency value in comparison to the other routing scheme i.e LEACH routing scheme, Base Station Position routing scheme and EnergyBalanced routing scheme.

\section{REFERENCE:}

[1]. Shio kumar ,singh,MP singh, and DK singh. - A Survey of Energy- Efficient Hierarchical Cluster-Based Routing in Wireless SensorNetworks. Int. J. of Advanced Networking and Applications (2010), VOL: 02, Page: 570-580

[2] S. Jerusha, K. Kulothungan \& A. Kannan ,'Location Aware Cluster Based Routing In Wireless Sensor Networks, Int. J. of Computer \& Communication Technology ISSN: 0975-7449, Volume-3, Issue-5, 2012

[3] Inderjit S. Dhillon, Yuqiang Guan and Brian Kulis "Kernel kmeans, Spectral Clustering and Normalized Cuts" ACM,International Conference on knowledge discovery and data mining 2004.

[4] Veena, K.N., Vijaya Kumar, B.P.," Dynamic clustering for Wireless Sensor Networks: A Neuro-Fuzzy technique approach"International Conference on Computational Intelligence and Computing Research (ICCIC), No. 1-6, IEEE 2010.

[5] W. B. Heinzelman, A. P. Chandrakasan, and H. Balakrishnan, "An application-specific protocol architecture for wireless sensor networks," IEEE Trans. Wireless Commun., vol. 1, no. 4, pp. 660-670, Oct. 2002.

[6] I. K. Samaras and G. D. Hassapis, "A modified DPWS protocol stack for 6LoWPAN-based wireless sensor networks," IEEE Trans. Ind. Inf., vol. 9, no. 1, pp. 209-217, Feb. 2013.

[7] R.K. Tripathi Y.N. Singh and N.K. Verma, Department of Electrical Engineering, Indian Institute of Technology, Kanpur, Kanpur 208 016, India,"Two-tiered wireless sensor networks - base station optimal positioning case study", in IET Wirel. Sens. Syst., 2012, Vol. 2, Iss. 4, pp. 351-360, doi: 10.1049/ietwss.2011.0152.

[8] Degan Zhang, Member, IEEE, Guang Li, Member, IEEE, Ke Zheng, Member, IEEE, Xuechao Ming, Member, IEEE, and Zhao-Hua Pan, Member, IEEE,"An Energy- Balanced Routing Method Based on Forward-Aware Factor for Wireless Sensor Networks", IEEE Transactions On Industrial Informatics, Vol. 10, No. 1, February 2014. 